\title{
SYNCHRONY ANALYSIS OF PAROXYSMAL GAMMA WAVES IN MEDITATION EEG
}

\author{
Jing Jin ${ }^{a}$, Justin Dauwels ${ }^{a}$, François B. Vialatte ${ }^{b}$,Andrzej Cichocki ${ }^{c}$ \\ ${ }^{a}$ Nanyang Technological University, School of Electrical and Electronic Engineering, Singapore 639798 \\ ${ }^{b}$ ESPCI ParisTech, Laboratoire SIGMA, 75231 Paris Cedex 05, France \\ ${ }^{c}$ RIKEN Brain Science Institute, Laboratory for Advanced Brain Signal Processing, Wako-Shi, Saitama, 3510198, Japan
}

\begin{abstract}
Mediation is a fascinating topic that is still relatively poorly understood. To investigate its physiological traits, electroencephalograms (EEG) were recorded during meditation sessions. In a recent study, paroxysmal gamma waves (PGWs) have been discovered in EEG of meditators practicing Bhramari Pranayama (BhPr). In this paper, the synchrony between those PGWs is investigated, revealing functional connectivity patterns in the brain during BhPr. Specifically, the method of Stochastic Event Synchrony (SES) is applied to pairs of PGW sequences in order to assess their synchrony. From those pairwise synchrony measures, large-scale functional connectivity patterns are extracted.

Three subjects possessing different levels of expertise in Bhramari Pranayama are considered. Strong synchrony can be observed in the temporal lobes for all 3 subjects, in addition to long-range interhemispheric connections. Consistent connectivity patterns are present for exhalation periods, while those patterns are substantially less stationary for inhalation periods. Interestingly, the synchrony seems to increase gradually during the meditation session. Moreover, the distribution of synchrony values seems to depend on the level of expertise in practicing BhPr: the higher the expertise, the more concentrated the intensity values.
\end{abstract}

Index Terms - Paroxysmal gamma wave; Electroencephalogram; Meditation; Bhramari Pranayama; Stochastic Event Synchrony

\section{INTRODUCTION}

Meditation refers to a broad variety of practices to train attention and awareness, so as to bring mental processes under greater voluntary control. Bhramari Pranayama (BhPr) is a yoga type meditation focusing on breathing, with alternative long exhalation periods and short inhalation periods. Regarding the benefit, BhPr may help to ease up the hormonal imbalance manifestation, such as hypertension, anxiety, and abnormal blood pressure [1]. Neuroimaging studies have been conducted to investigate the brain activity during meditation. Several fMRI studies demonstrated that the temporal lobes are active during meditation [2, 3, 4]. Enhanced activity in gamma band EEG during meditation has also been reported [5, 6, 7]; in particular, subjects exhibit paroxysmal gamma waves (PGWs) in the temporal lobes and some other brain areas. In earlier work [8], we proposed techniques to extract PGWs from BhPr EEG in an automated fashion. In this paper, we build upon our earlier work, and analyze the synchrony between PGWs occurring at different EEG channels, leading to functional connectivity patterns in the brain during BhPr meditation. Concretely, we apply the "Stochastic Event Synchrony" (SES) method [9] to quantitatively assess the similarity of pairs of PGW sequences. We aim to reveal the functional connections between brain regions during BhPr. As in [8], we consider three subjects possessing different levels of expertise in $\mathrm{BhPr}$ (beginner, intermediate, and expert).

Earlier studies on meditation EEG have reported enhanced long-range EEG coherence, suggesting stronger coordination of brain activity. For instance, studies of transcendental meditation (TM) demonstrated enhanced EEG coherence during practice of TM, along with synchronized alpha power in the frontal cortex [10,11, 12]. High-amplitude gamma-band oscillations and long-range phase-synchrony were observed as well in the EEG of long-term Buddhist meditators [7, 13]. Along those lines, the study at hand explores synchrony in the gamma band, between PGWs occurring in different brain regions.

This paper is organized as follows. In Section 2, we elaborate on our experimental data, and briefly review the techniques for automated PGW extraction; we outline the "Stochastic Event Synchrony" (SES) method [9] for quantifying synchrony of 1-D point processes (PGWs in particular). In Section 3, we present our numerical results. In Section 4, we offer concluding remarks.

\section{METHODS}

\subsection{Bhramari Pranayama EEG Data}

We analyze scalp EEG recorded from three subjects practicing BhPr meditation; the same data was also analyzed in $[5,8]$. The three subjects possess different levels of ex- 
pertise practicing BhPr. The beginner " $B$ " had no experience before; the intermediate " $I$ " was trained for a month; and the expert " $E$ " was trained at least for 4 months. EEG was recorded during several BhPr sessions, in which each subject performed approximately 20 breathing episodes. During each exhalation period, the subject produced a typical humming bee sound. An ambient sound signal was recorded simultaneously to differentiate each breathing period. The EEG recordings were conducted in an electrically shielded room using a Biosemi system with 128 active electrodes. The sampling rate was $2048 \mathrm{~Hz}$ and an analog filter between 1 and 300 $\mathrm{Hz}$ was applied. Moreover, notch filters were applied at 50 $\mathrm{Hz}$ and every harmonic of $25 \mathrm{~Hz}$ to remove the power line interferences.

\subsection{Automated Paroxysmal Gamma Wave Detection}

The schematic diagram of the automated procedure proposed in [8] for PGW extraction is depicted in Fig. 1. First, principal component analysis (PCA) [14] is applied, to reduce the number of channels. Let $Z \in \mathbb{R}^{N \times M}$ denote the EEG recording consisting $N$ channels, with $M$ samples each. Denoting the eigenvalues of the covariance $R$ of $Z$ by $\lambda_{1}, \lambda_{2}, \cdots, \lambda_{N}$ in descending order and the corresponding eigenvectors by $u_{1}, u_{2}, \cdots, u_{N}, Z$ can be compressed by retaining the top $L$ (with $L \ll N$ ) dimensions. The compressed data $Y \in \mathbb{R}^{L \times M}$ can be obtained as:

$$
Y=\left[\begin{array}{llll}
u_{1} & u_{2} & \cdots & u_{L}
\end{array}\right]^{T} Z .
$$

The parameter $L$ is chosen such that:

$$
\frac{\sum_{i=1}^{L} \lambda_{i}}{\sum_{i=1}^{N} \lambda_{i}} \geq \zeta
$$

with $\zeta \in(0,1]$ (we choose $\zeta=0.95)$.

Next, blind signal separation (BSS) [15] is applied to identify the sources from the compressed EEG. Our hypothesis is that the compressed data $Y$ in (1) is a linear combination of independent sources $X \in \mathbb{R}^{L \times M}$ located in the brain, i.e., $Y=A X+N$, with $A \in \mathbb{R}^{L \times L}$ the mixing matrix and $N \in \mathbb{R}^{L \times M}$ the i.i.d. Gaussian noise. To find $X$, it is cast as an inverse problem $X=W Y$, where the weight $W \in \mathbb{R}^{L \times L}$ is unknown. BSS method "Second Order Blind Identification" (SOBI) [16] is applied to infer this weight matrix $W$ by:

$$
\hat{W}=\hat{U}^{H}\left[\left(\lambda_{1}-\hat{\sigma}^{2}\right)^{-0.5} u_{1}, \cdots,\left(\lambda_{L}-\hat{\sigma}^{2}\right)^{-0.5} u_{L}\right]^{H},
$$

where $\hat{\sigma}^{2}$ is the estimated noise variance, computed using resting EEG. The unitary matrix $\hat{U}$ is obtained as joint diagonalizer of the sample estimates.

We developed a morphological filter in [8] to estimate the PGW rate for each source, as we will explain in more detail

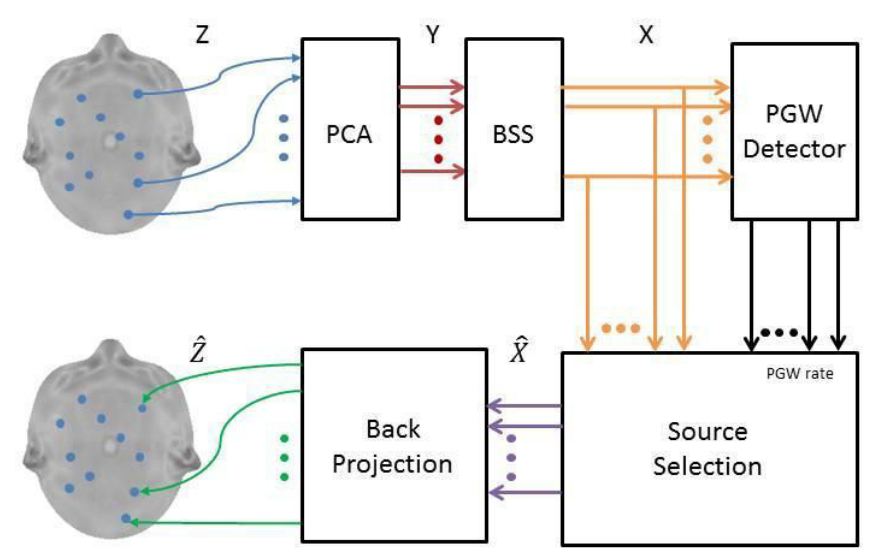

Fig. 1: Automated extraction of PGWs [8].

in the following. We then select the sources with a relatively large number of PGWs. Lastly, the signals from these selected sources are back-projected in sensor domain, in order to reconstruct 128-channel EEG data.

By definition, the paroxysmal gamma waves (PGWs) in meditation EEG are characterized by distinct high-frequency biphasic patterns, with a shape close to an inter-ictal spike [5] (see Fig. 2). The automated PGW detection method that we proposed in [8] exploits the morphological characteristics of PGWs, as illustrated in Fig. 2.

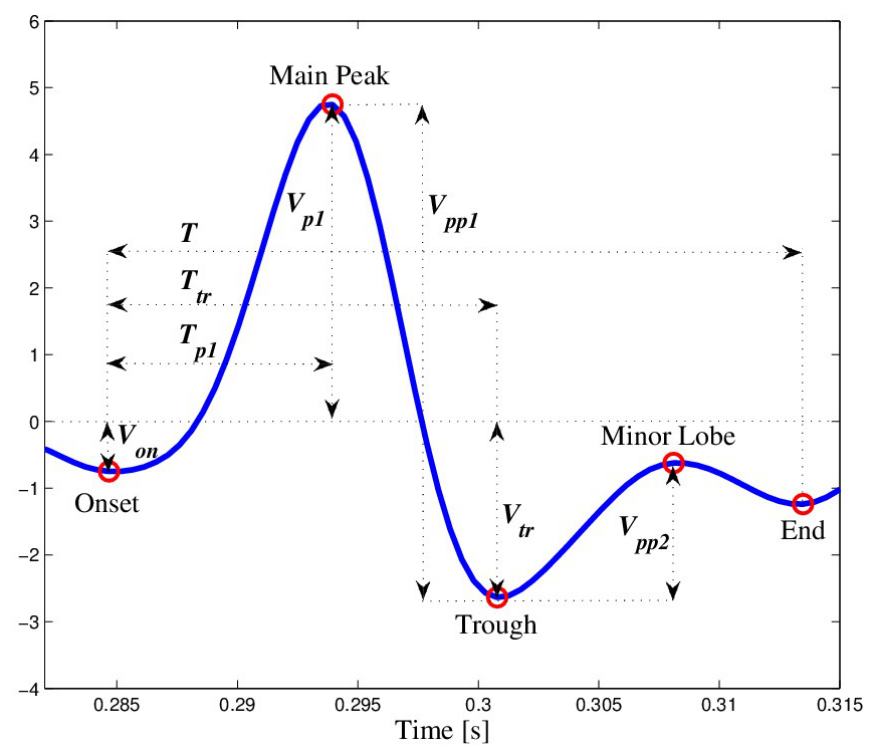

Fig. 2: Morphological parameters of a PGW [8].

Specifically, it follows the rules: $(i)$ the peak value $V_{p 1}$ of the main peak must be larger than multiple standard deviation of the signal, i.e., $V_{p 1}>\alpha \sigma_{x}$, with $\sigma_{x}$ the standard deviation of the entire signal and $\alpha$ a scalar; ( $i i)$ the second peak $V_{p p 2}$ must be significantly smaller than the main peak $V_{p p 1}$, i.e., $\rho_{1} V_{p p 1} \leq V_{p p 2} \leq \rho_{2} V_{p p 1}$ with $\rho_{1}<\rho_{2}<1$; (iii) the 
trough should be below the onset point, i.e., $V_{o n}>V_{t r}$; and finally $(i v)$ the duration $T$ of the PGW should be smaller than a constant $\tau$. The morphological parameters $\left(\alpha, \rho_{1}, \rho_{2}, \tau\right)$ are derived from "true" PGW samples manually extracted from each individual channel (or source). For each PGW, the parameters $\left(\alpha, \rho_{1}, \rho_{2}, \tau\right)$ are computed, and extreme values $\left(\alpha_{\min }, \rho_{1 \min }, \rho_{2 \max }, \tau_{\max }\right)$, are kept as the final representative for a particular channel (or source).

We have validated the performance of this method by comparison with two other methods for spike detection. As investigated in [8], our method vastly outperforms those two methods in terms of sensitivity and false alarm rate. It is computational effective and reliable in detection of PGW.

In this paper, we apply the automated method in [8] to remove artifacts, and extract components (sources) with a relatively large number of PGWs. Those sources are then backprojected to sensor domain yielding artifact-free EEG containing PGWs. Finally, the PGW sequences are analyzed by the SES method to construct functional connectivity patterns during BhPr.

\subsection{Stochastic Event Synchrony}

The "Stochastic Event Synchrony"(SES) measures [9] assesses the similarity of pairs of point processes. The PGW sequences extracted form each EEG channel can be viewed as point processes, where each PGW is considered as an event (point). Unlike classical similarity measures for point processes [9], SES quantifies the alignment of two point processes using multiple parameters: time delay $\delta_{t}$, variance of the time jitter $s_{t}$, and fraction of coincident events $\gamma$. SES captures two different aspects of synchrony: timing precision and reliability, quantified by $s_{t}$ and $\gamma$ respectively. Two point processes are considered synchronous if they are identical apart from: $(i)$ a time delay $\delta_{t} ;(i i)$ small deviations in the event occurrence times; and ( iii) a few event insertions and/or deletions. More precisely, the event timing jitter should be significantly smaller than the average inter-event time; and the number of deletions and insertions should comprise only a small fraction of the total number of events, corresponding to a large coincidence rate $\gamma$.

The SES parameters are computed via statistical inference in a generative model. The latter can be explained by the symmetric procedure shown in Fig. 3 for generating two point processes $x$ and $x^{\prime}$ with length $n$ and $n^{\prime}$ respectively. First, a hidden point process $v$ of length $\ell$ is generated, with events $v_{k}$ mutually independent and uniformly distributed in $\left[0, T_{0}\right]$. The point process $z$ and $z^{\prime}$ are obtained by shifting $v$ over $\pm 0.5 \delta_{t}$ respectively, and slightly perturbing the timing with variance $0.5 s_{t}$. Finally, $x$ and $x^{\prime}$ are derived from $z$ and $z^{\prime}$ by deleting events with probability $p_{d}$. $\quad b$ and $b^{\prime}$ are binary strings indicating whether the events in $x$ and $x^{\prime}$ are coincident/paired $\left(b_{k}=1\right.$ if $x_{k}$ is non-coincident, $b_{k}=0$ otherwise; likewise for $b_{k}^{\prime}$ ). As was shown in [9], this procedure can be

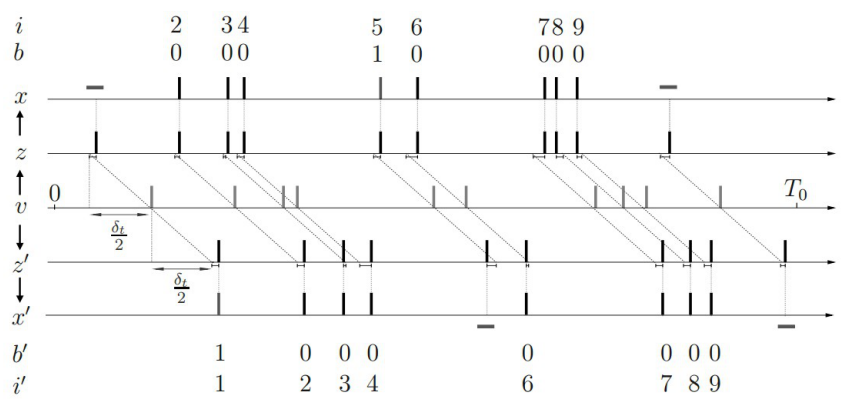

Fig. 3: Symmetric procedure relating $x$ and $x^{\prime}$ [9].

summarized by the generative statistical model:

$$
p\left(x, x^{\prime}, b, b^{\prime}, v, \delta_{t}, s_{t}, \ell\right) \propto \beta^{n_{d e l}^{t o t}} \prod_{k=1}^{n_{c o}^{t o t}} \mathcal{N}\left(x_{j_{k}}^{\prime}-x_{j_{k}} ; \delta_{t}, s_{t}\right)
$$

with $\left(x_{j k}, x_{j_{k}^{\prime}}^{\prime}\right)$ pairs of coincident events, $n_{c o}^{t o t}=n+n^{\prime}-\ell$ the total number of coincident event pairs, and $\beta$ as a function of $p_{d}$ and $\ell$. The variables in that model are inferred by cyclic maximization [9], resulting in estimates of the pairs of coincident events $\left(x_{j k}, x_{j_{k}^{\prime}}^{\prime}\right)$. From the latter, the parameters $\delta_{t}, s_{t}$ and $\gamma$ can directly be computed.

\section{RESULTS AND DISCUSSION}

In this paper, SES parameters $\gamma$ and $\sigma_{t}=\sqrt{s_{t}}$ are used to study the synchrony of PGW sequences. We define the strength of connection between each pair of channels (brain locations) by $\gamma$, the faction of coincident events. To infer the brain networks, only significantly strong connections will be selected. Both SES parameters and PGW count are considered in the selection process: We only keep connections with $\gamma>0.75$, $\sigma_{t}<0.1 T_{\mathrm{isi}}$, and pairs of channels with PGW rate greater than the 0.95 percentile of all the channels. We apply those rules to all three subjects.

The results of SES analysis are shown in Fig. 4. For each subject, there are two rows of figures: The top row depicts the normalized power distribution, reflecting the brain activities during the different breathing episodes (either exhalations or inhalations); the bottom figures display the functional connectivity maps, where the connections are selected according to the criteria mentioned earlier. The strength of the connections is defined by $\gamma$, and are color coded. The PGW rate distribution is displayed in the background to visualize the corresponding active brain regions. For the sake of simplicity, only colorless contours of the PGW rate distribution are shown here.

Strong synchrony can be found in temporal lobes for all three subjects. In addition, consistent patterns of connections are present for exhalation periods, while it is relatively nonstationary for inhalation periods. Interestingly, long-range 


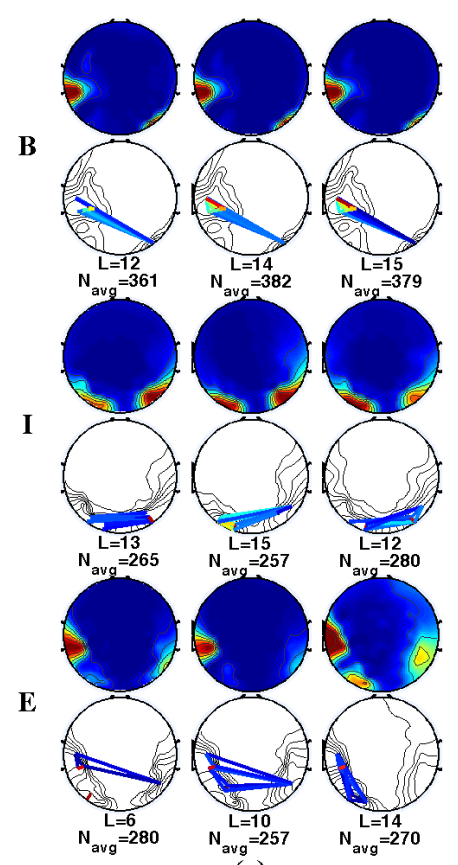

(a)

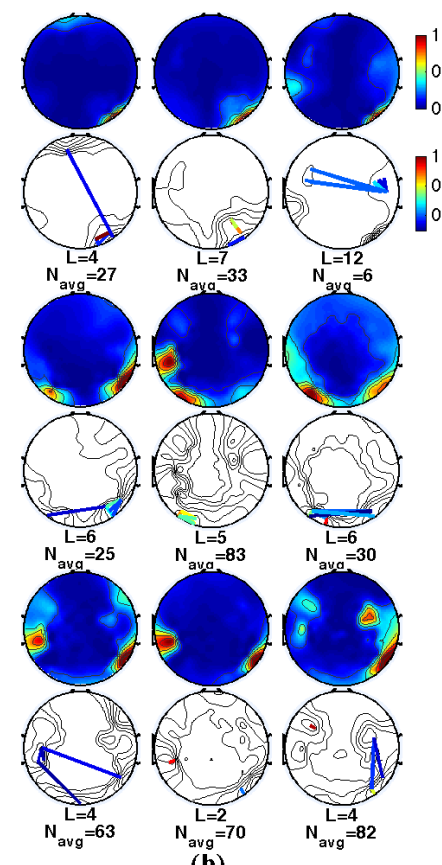

(b)

Fig. 4: Power distribution and connectivity networks with connection strength $\gamma$ for three subjects $(B, I$, and $E$ ) and three successive periods of (a) exhalations, and (b) inhalations. $L$ is the total number of connections, and $N_{\text {avg }}$ is the average PGW count of each connection.
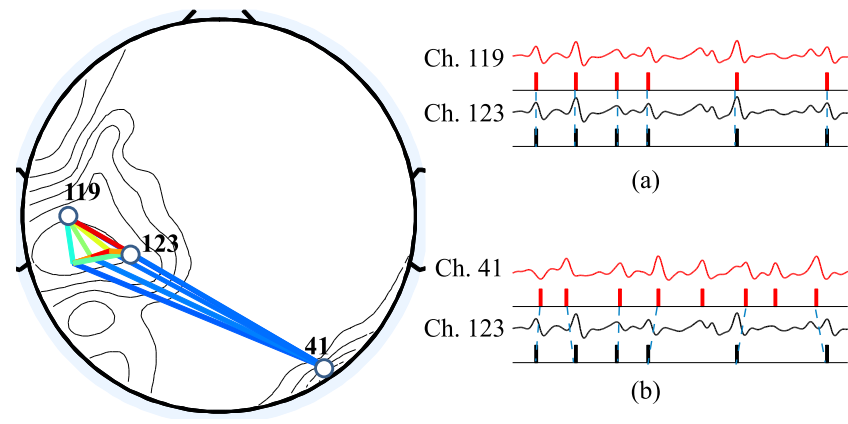

(a)

Fig. 5: Connectivity map (left) and EEG (right) of B3 with extracted PGW sequences from a pair of (a) adjacent channels Ch. 119 and Ch. 123 with $\left(\gamma, \sigma_{t}, \delta_{t}\right)=$ $\left(97.3 \%, \ll 0.01 T_{\mathrm{isi}}, 0 s\right)$; and (b) distant channels Ch. 41 and Ch. 123 with $\left(\gamma, \sigma_{t}, \delta_{t}\right)=\left(79.6 \%, 0.01 T_{\text {isi }}, 10 m s\right)$.

connections between distinct active brain regions are found for all three subjects with moderate strength (see Fig. 4). As an illustration, in Fig. 5 we show PGW sequences extracted from local and distant pairs of channels. Clearly, there seems to be synchrony even between distant channels. The observed large-scale synchrony creates perhaps more coordinated and effective brain activations.

Another fascinating observation concerns the change in synchrony during the meditation session. As shown in Fig. 4a, the number of connections and their strength tend to increase

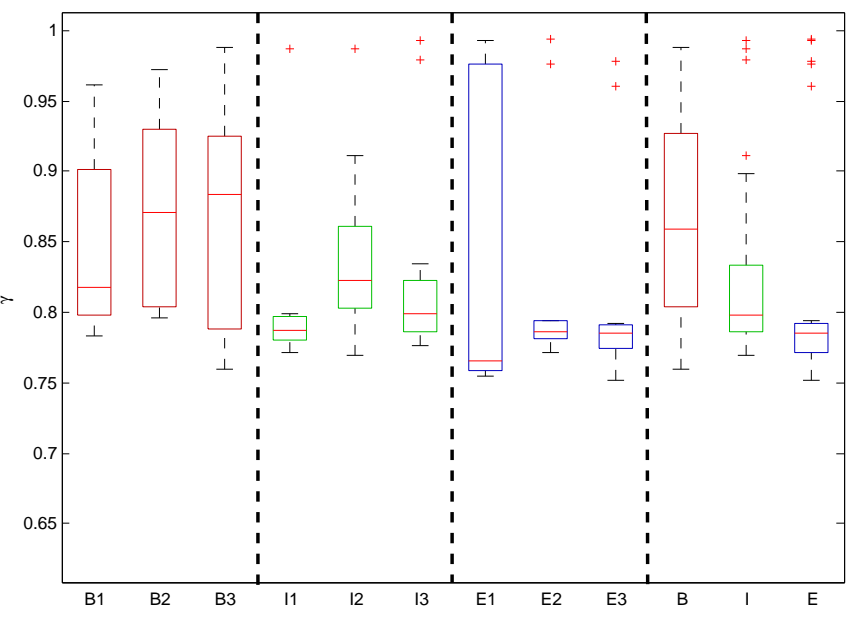

Fig. 6: Synchrony measure $\gamma$ for the three subjects. First three groups from left: Boxplot of $\gamma$ for three successive exhalation periods for $B, I$ and $E$. Right: Boxplot of $\gamma$ for the three subjects (jointly for the three exhalation periods).

over time, especially for $B$ (see Fig. 6 left). For each subject, the median of $\gamma$ gradually increases along the time. The distribution of the connection strength $\gamma$ depends on the level of expertise of practicing $\mathrm{BhPr}$ : the higher the expertise, the more concentrated the strength values (see Fig. 6 right). Intuitively, compared to a novice, it may be easier for more experienced meditators to settle down into the cognitive state of meditation.

\section{CONCLUSIONS}

We investigated the synchrony of paroxysmal gamma waves (PGWs) in electroencephalograms (EEG) recorded during Bhramari Pranayama (BhPr) meditation. Three subjects with different levels of expertise in BhPr (beginner, intermediate, and expert) are considered. To quantify the synchrony of the PGWs, we applied the "Stochastic Event Synchrony" (SES) method to pairs of PGW sequences extracted from the EEG. Specifically, the fraction $\gamma$ of coincident PGWs is used as the strength of the each connection.

Strong synchrony can be observed in the temporal lobes for all three subjects, in addition to long-range interhemispheric connections. Consistent connectivity patterns are present for exhalation periods, while those patterns are substantially more non-stationary for inhalation periods. Interestingly, the synchrony increases gradually along the time during meditation. Moreover, the distribution of synchrony values depends on the level of expertise in practicing BhPr: the higher the expertise, the more concentrated the synchrony values.

In the future, we plan to apply multi-dimensional SES to PGW sequences, which may provide additional insights into the synchrony measure. 


\section{REFERENCES}

[1] S. Pandey, N. Mahato, and R. Navale, "Role of selfinduced sound therapy: Bhramari pranayama in tinnitus," Audiological Medicine, vol. 8, no. 3, pp. 137-141, 2010.

[2] S. Lazar, G. Bush, R. Gollub, G. Fricchione, G. Khalsa, and $\mathrm{H}$. Benson, "Functional brain mapping of the relaxation response and meditation," Neuroreport, vol. 11, no. 7, pp. 1581-1585, 2000.

[3] B. Hölzel, U. Ott, H. Hempel, A. Hackl, K. Wolf, R. Stark, and D. Vaitl, "Differential engagement of anterior cingulate and adjacent medial frontal cortex in adept meditators and non-meditators," Neuroscience letters, vol. 421, no. 1, pp. 16-21, 2007.

[4] B. K. Hölzel, U. Ott, T. Gard, H. Hempel, M. Weygandt, K. Morgen, and D. Vaitl, "Investigation of mindfulness meditation practitioners with voxel-based morphometry," Social Cognitive and Affective Neuroscience, vol. 3, no. 1, pp. 55-61, 2008.

[5] F. Vialatte, H. Bakardjian, R. Prasad, and A. Cichocki, "Electroencephalographic paroxysmal gamma waves during bhramari pranayama: A yoga breathing technique," Consciousness and cognition, vol. 18, no. 4, pp. 977-988, 2009.

[6] D. Lehmann, P. Faber, P. Achermann, D. Jeanmonod, L. Gianotti, and D. Pizzagalli, "Brain sources of eeg gamma frequency during volitionally meditationinduced, altered states of consciousness, and experience of the self," Psychiatry Research: Neuroimaging, vol. 108, no. 2, pp. 111-121, 2001.

[7] A. Lutz, L. Greischar, N. Rawlings, M. Ricard, and R. Davidson, "Long-term meditators self-induce highamplitude gamma synchrony during mental practice," Proceedings of the National Academy of Sciences of the United States of America, vol. 101, no. 46, pp. 1636916373, 2004.

[8] M. Vazquez, J. Jing, J. Dauwels, and F. Vialatte, "Automated detection of paroxysmal gamma waves in meditation eeg," in Acoustics, Speech, and Signal Processing, ICASSP, IEEE International Conference, IEEE, 2013.

[9] J. Dauwels, F. Vialatte, T. Weber, and A. Cichocki, "Quantifying statistical interdependence by message passing on graphs part i: One-dimensional point processes," Neural Computation, vol. 31, no. 8, pp. 21522202, 2009.

[10] M. C. Dillbeck, D. W. Orme-Johnson, and R. K. Wallace, "Frontal eeg coherence, h-reflex recovery, concept learning, and the tm-sidhi program," International Journal of Neuroscience, vol. 15, no. 3, pp. 151-157, 1981.

[11] F. Travis and R. K. Wallace, "Autonomic and eeg patterns during eyes-closed rest and transcendental meditation (tm) practice: the basis for a neural model of tm practice," Consciousness and cognition, vol. 8, no. 3, pp. 302-318, 1999.

[12] R. Hebert, D. Lehmann, G. Tan, F. Travis, and A. Arenander, "Enhanced eeg alpha time-domain phase synchrony during transcendental meditation: Implications for cortical integration theory," Signal Processing, vol. 85, no. 11, pp. 2213-2232, 2005.

[13] R. J. Davidson and A. Lutz, "Buddha's brain: Neuroplasticity and meditation [in the spotlight]," Signal Processing Magazine, IEEE, vol. 25, no. 1, pp. 176-174, 2008.

[14] I. S. Lindsay, "A tutorial on principal components analysis," Ithaca: Cornell University, pp. 26-28, 2002.

[15] J. Cardoso, "Blind signal separation: statistical principles," Proceedings of the IEEE, vol. 86, no. 10, pp. 2009-2025, 1998.

[16] A. Belouchrani, K. Abed-Meraim, J. Cardoso, and E. Moulines, "A blind source separation technique using second-order statistics," Signal Processing, IEEE Transactions on, vol. 45, no. 2, pp. 434-444, 1997. 